\title{
jardín infantil
}

F. M. GARCIA ORDOÑEZ y J. M. DEXEUS, arquitectos

$141 \cdot 77$
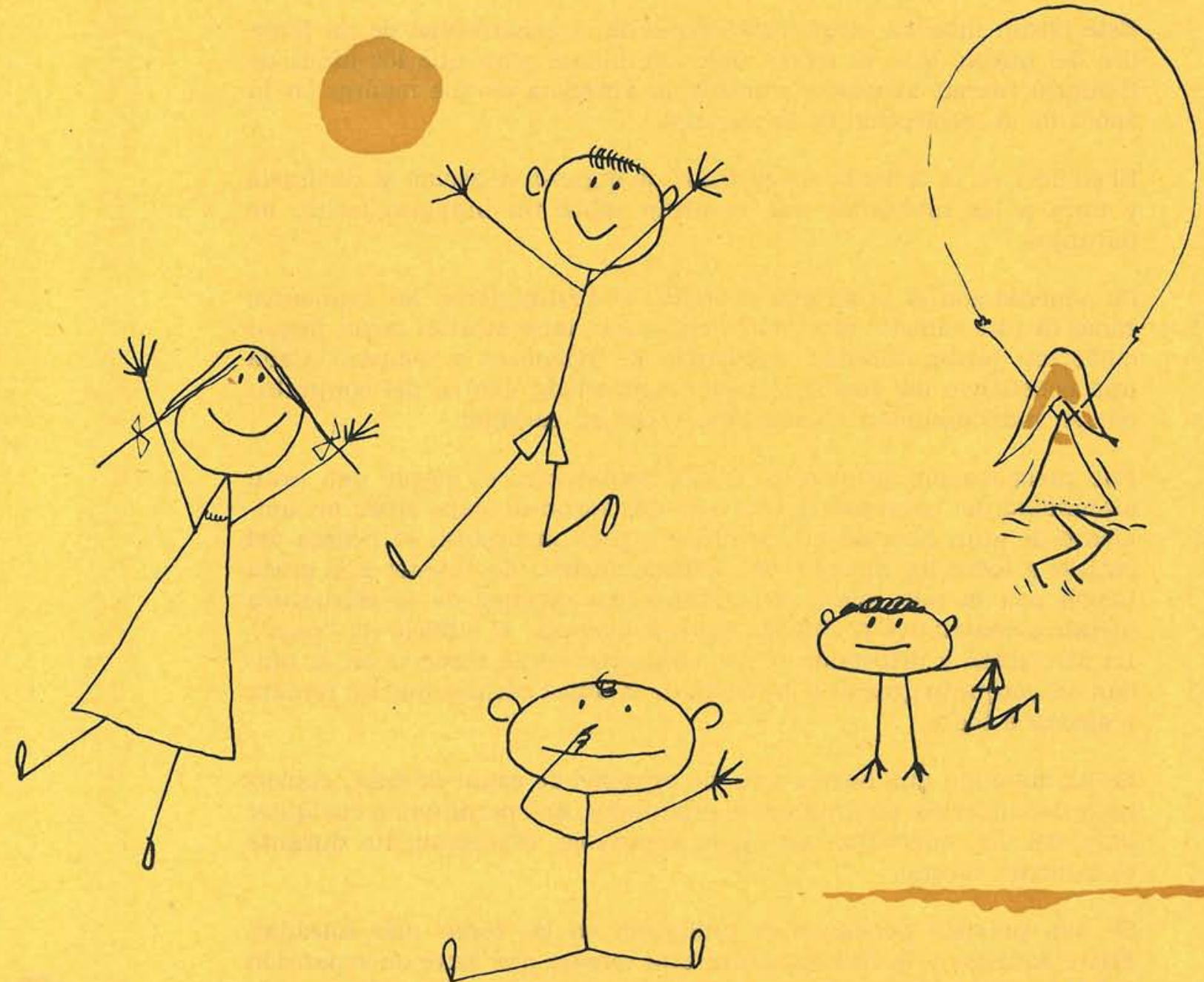

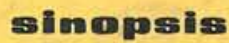

Este jardín infantil ha sido construido con el fin de que los niños de Beniarjó puedan ser atendidos, durante la ausencia de sus madres, en la época de la recolección de la naranja.

El edificio consta de las siguientes zonas: direcci6n, ocho aulas, laboratorios, aseos, juegos cubiertos, jardin interior, residencia de religiosas y ciaustro. Cada una constituye un elementivi bien diferenciado centro del conjunto, por s

Han sido cuidadosamente estudiadas la iluminación y ventilación de las diversas dependencias, $y$ se ha conseguido, felizmente, que el conjunto ofrezca una gran diafanidad, crear un ambiente de gran tad en los niños, e incorporar la belleza del paisaje a todos los rincones del edificio.

La estruetura es metálica y predomina el ladrillo visto, de color hueso, en las aulas y la tirolesa rústica en el pequeño convento. 
Beniarjó es un pueblecito situado a unos $70 \mathrm{~km}$ al sur de Valencia, en una zona de naranjos y con un grato clima mediterráneo. Atraviesa este vergel un simpático riachuelo procedente de las suaves montañas que se divisan a cierta distancia enriqueciendo con su presencia tan sin par paisaje, bañado, al atardecer, por el penetrante aroma del azahar.

Este jardín infantil surgió como fruto de la generosidad de un hombre del pueblo que, al morir, dejó sus bienes para que los niños de Beniarjó fueran atendidos durante la ausencia de sus madres en la época de la recolección de la naranja.

El edificio, en la fachada opuesta a la de ingreso, se asoma al riachuelo y mira a las montañas que se alzan sobre un inmenso jardín de naranjos.

De acuerdo con el programa previsto, se distinguieron las siguientes zonas de este edificio: dirección, ocho aulas, laboratorios, aseos, juegos cubiertos, jardín interior, residencia de religiosas y claustro. Cada una constituye un elemento bien diferenciado dentro del conjunto: por su individualidad volumétrica y por su carácter.

Fue preocupación primordial de los arquitectos el lograr una gran diafanidad del conjunto, y esto por dos razones: para crear un ambiente de gran libertad en los niños y para incorporar la belleza del paisaje a todos los rincones del edificio, además de obtener una grata fusión con la naturaleza circundante. La esbeltez de la estructura metálica contribuyó a obtener aquella ligereza: el edificio no "pesa". La diversidad y dispersión de los componentes se resuelve en la unidad del conjunto gracias a la fuerza de la única cornisa que los remata y abraza a todos.

Se ha obtenido una buena serie de espacios de estar exterior, cubiertos y descubiertos, distintamente orientados, que permiten, a cualquier hora del día, encontrar un lugar agradable, especialmente durante el caluroso verano.

Se han previsto persianas de guillotina en las zonas más soleadas. Entre aquéllas y la fachada corre una terraza que sirve de expansión exterior y de aislamiento.

Predomina el ladrillo visto, de color hueso, en las aulas, y la tirolesa rústica en el conventillo.

Cada patio o claustro está ambientado de acuerdo con la función del elemento o elementos que le circundan.

Las circulaciones entre los distintos elementos se acusan tanto en el pavimento como en el techo, colgando éste por debajo del resto y realzando aquél, como una bandeja, por encima de los demás y del jardín.

Se ha estudiado cuidadosamente la iluminación y ventilación de las aulas, que son ocho en total, con capacidad, cada una, para 30 niños.

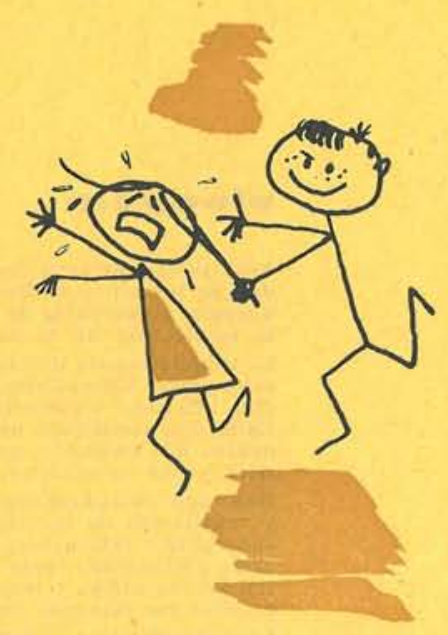



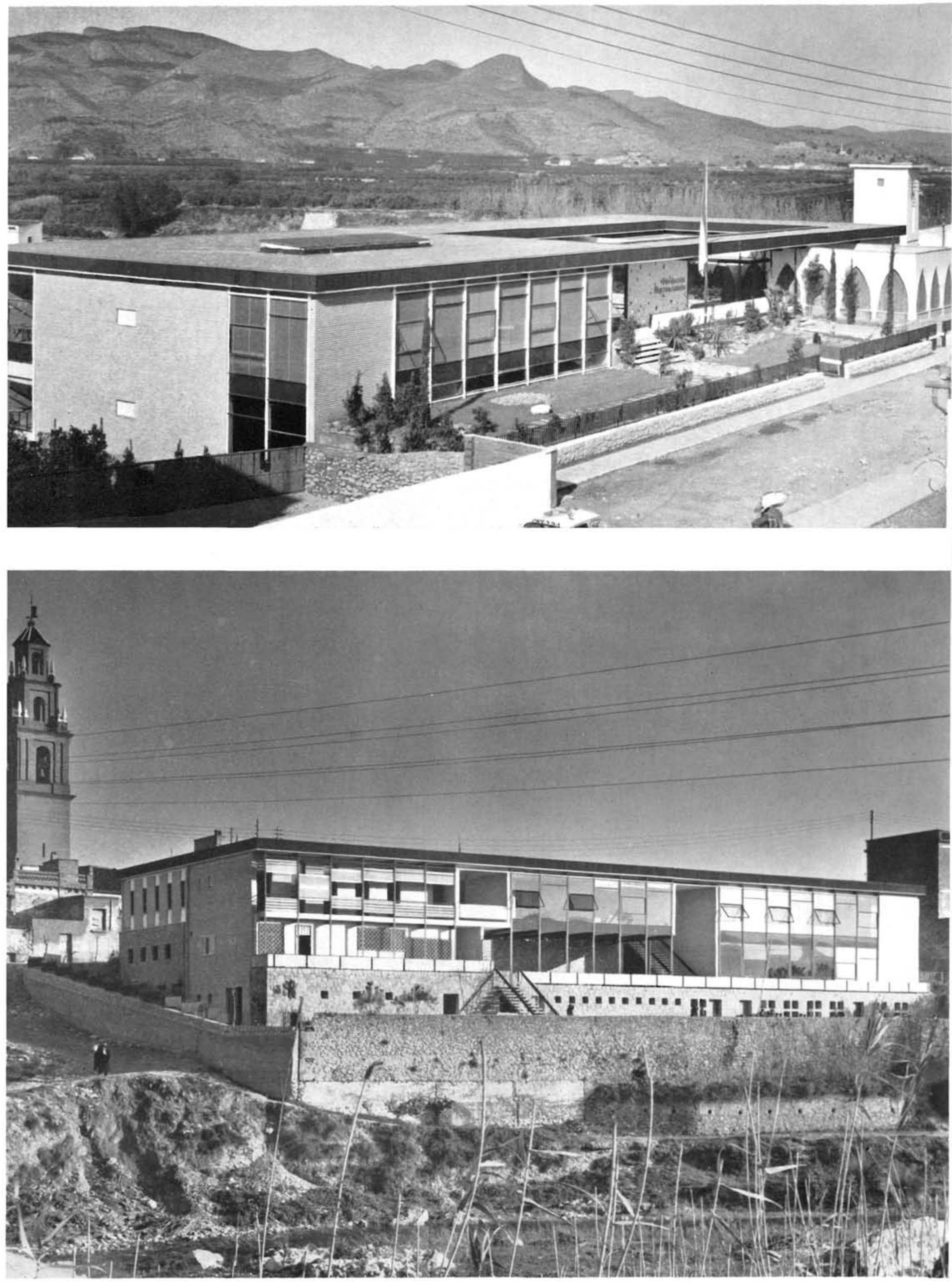


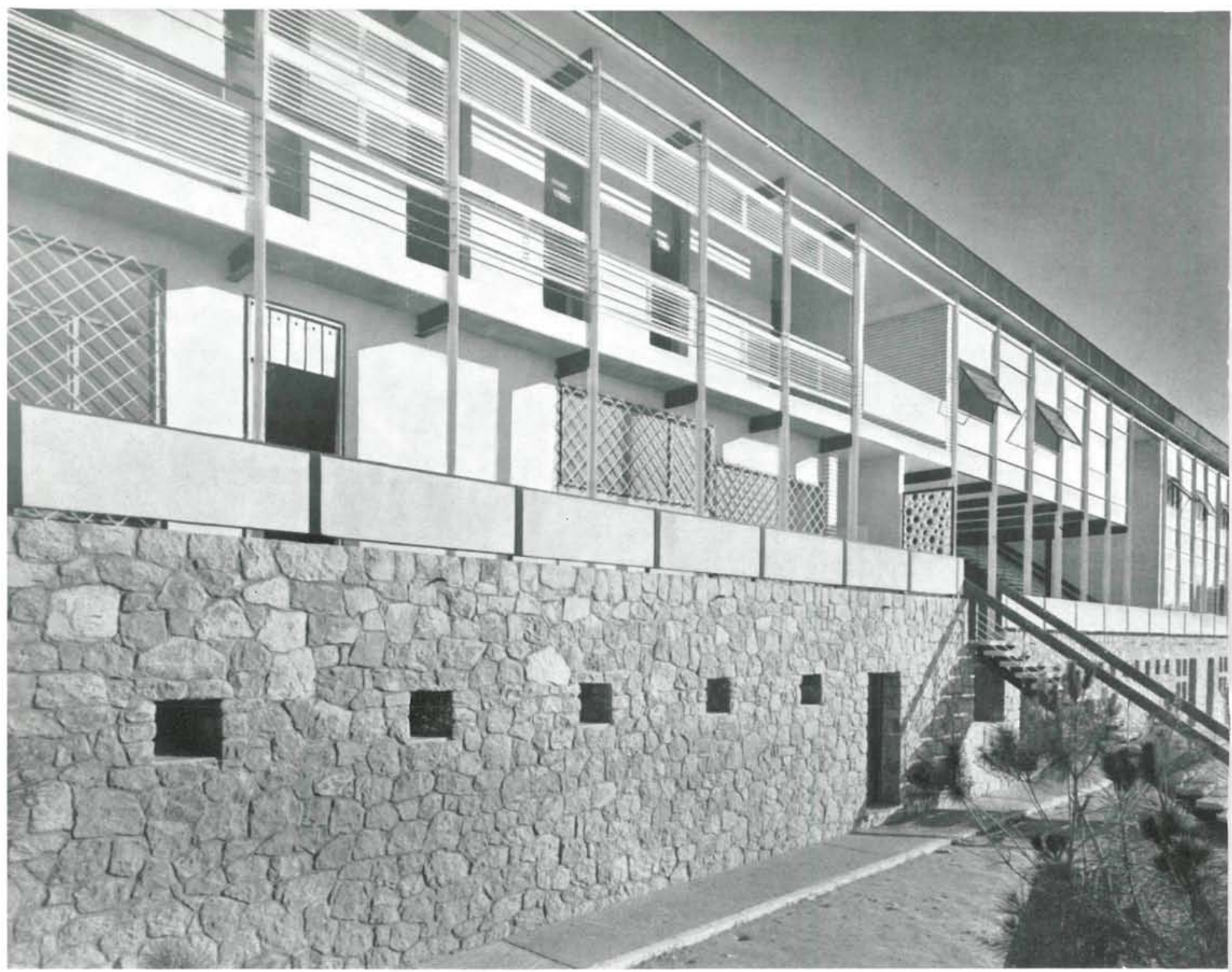

\section{cleterille de farthada posstererior}

Entrana

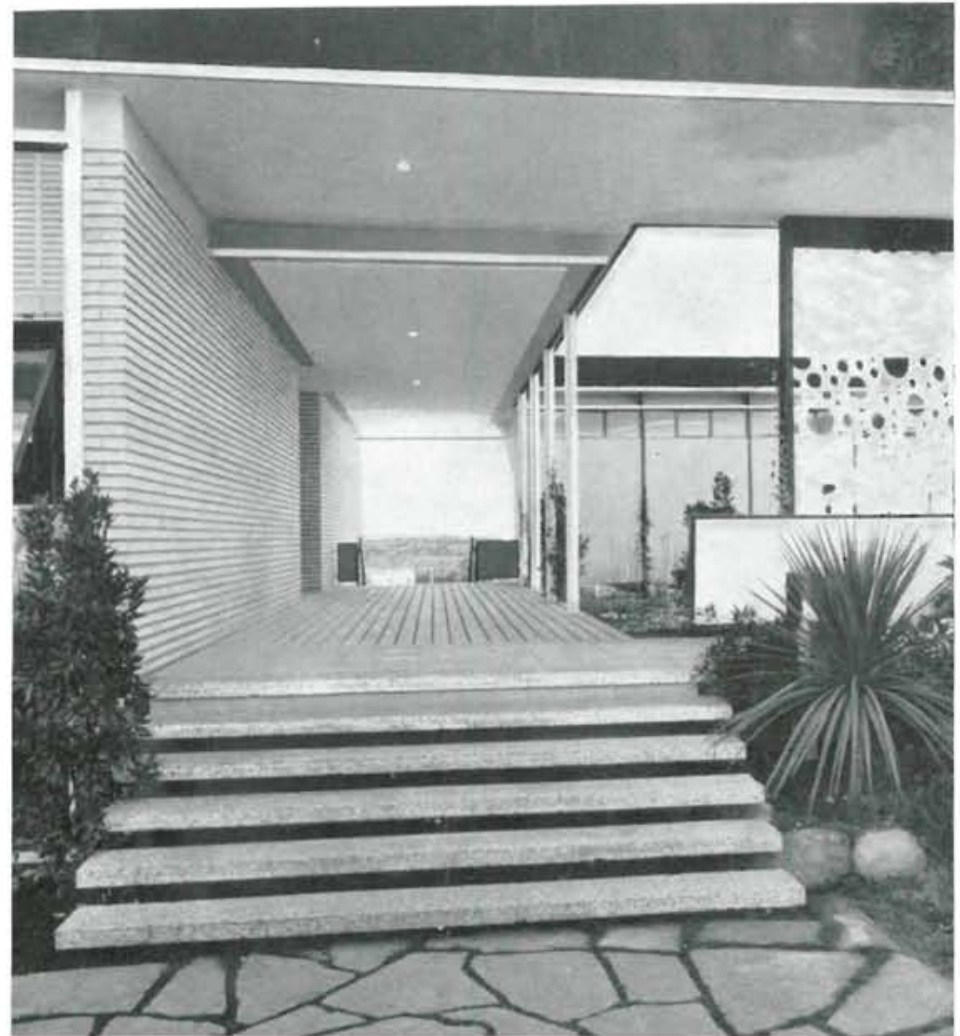



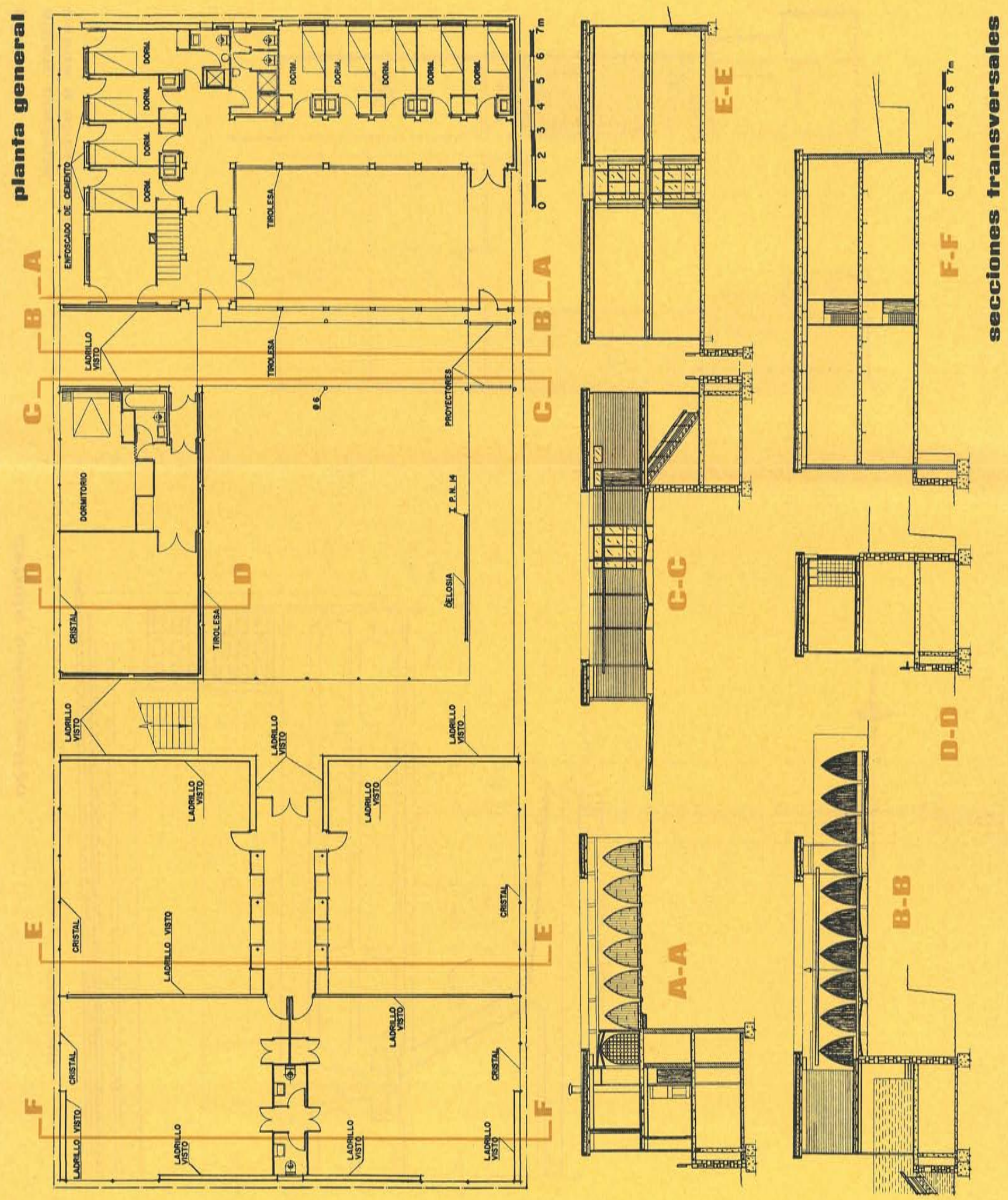


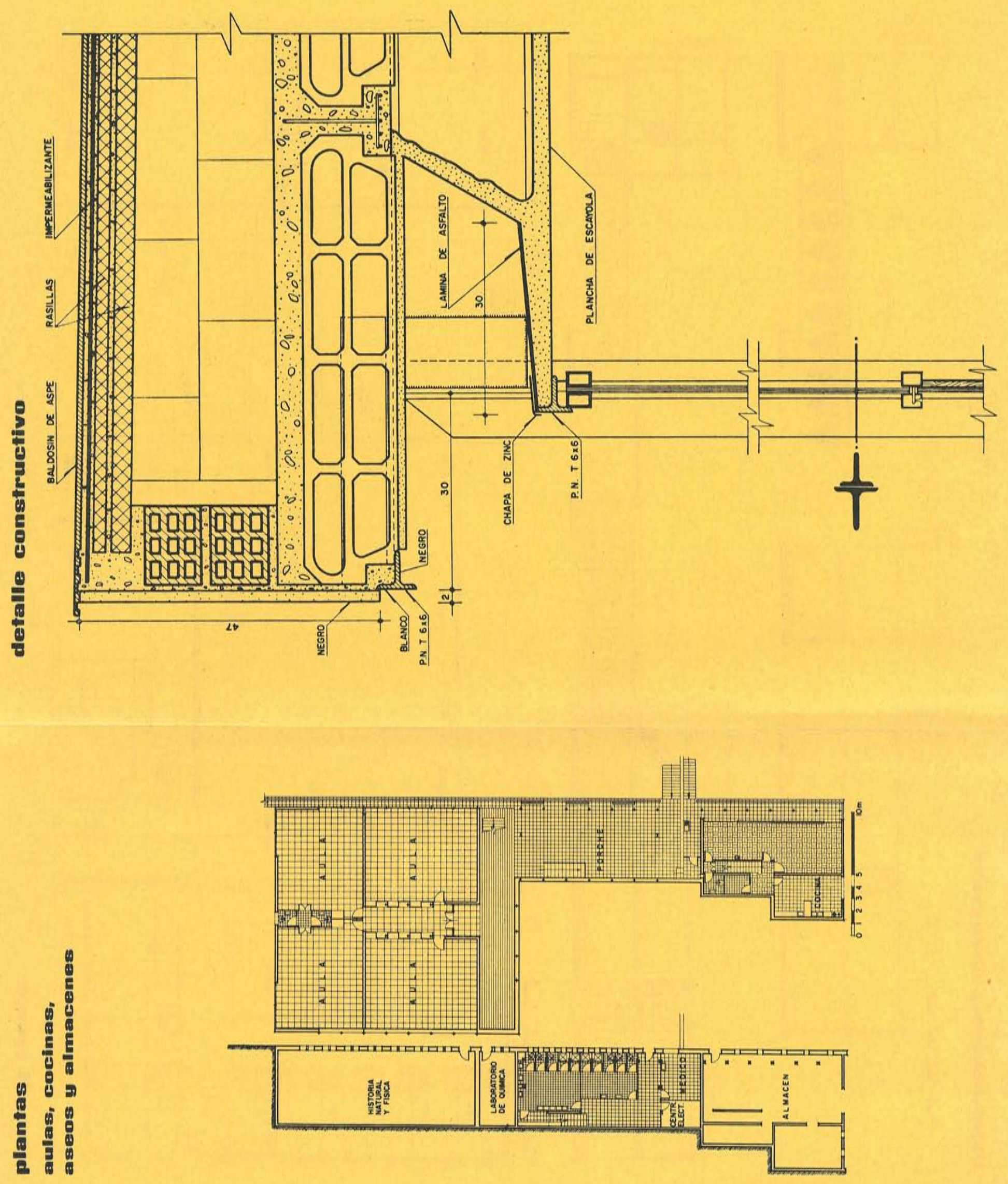



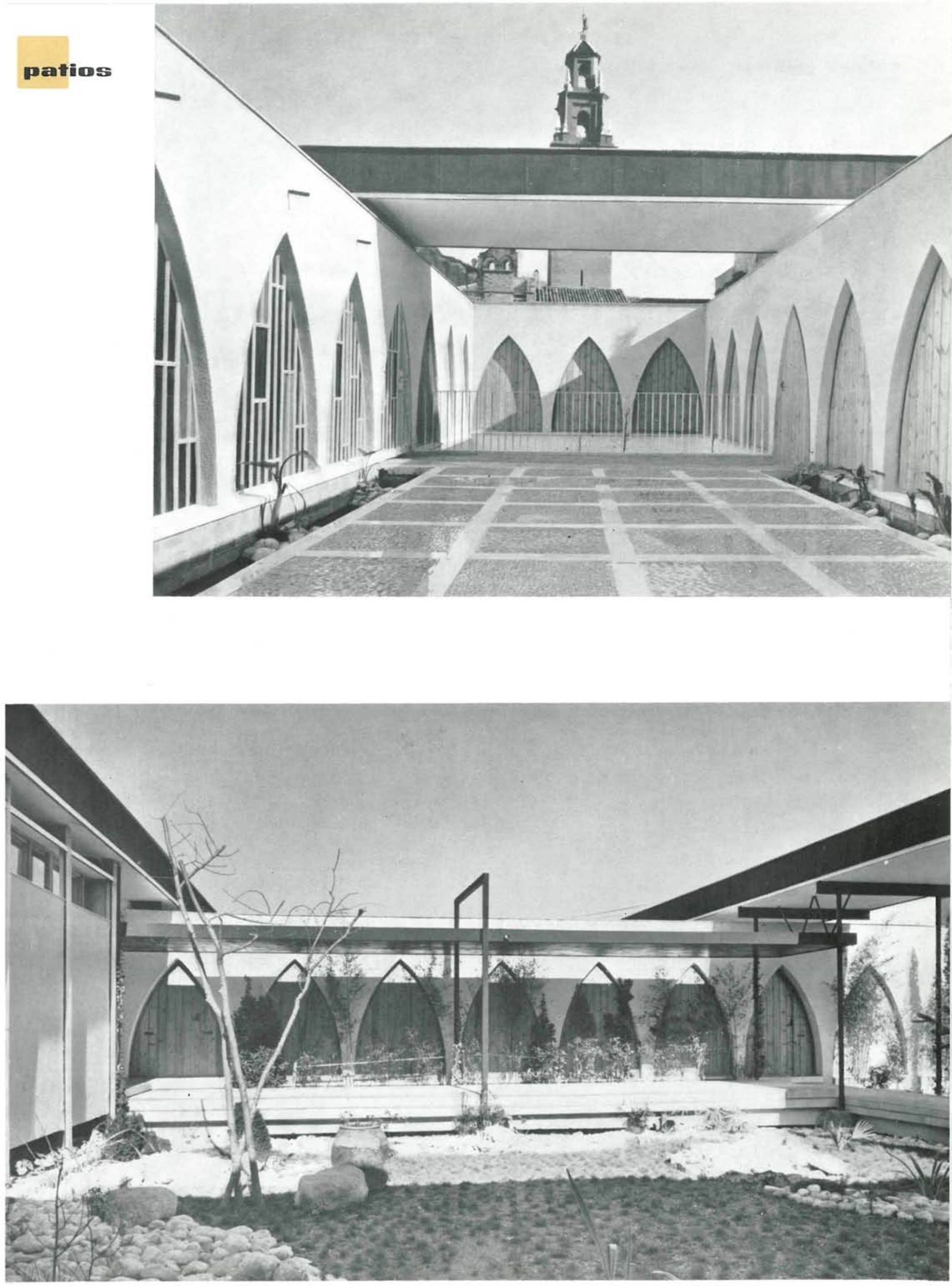
porcthe ale ruceesso
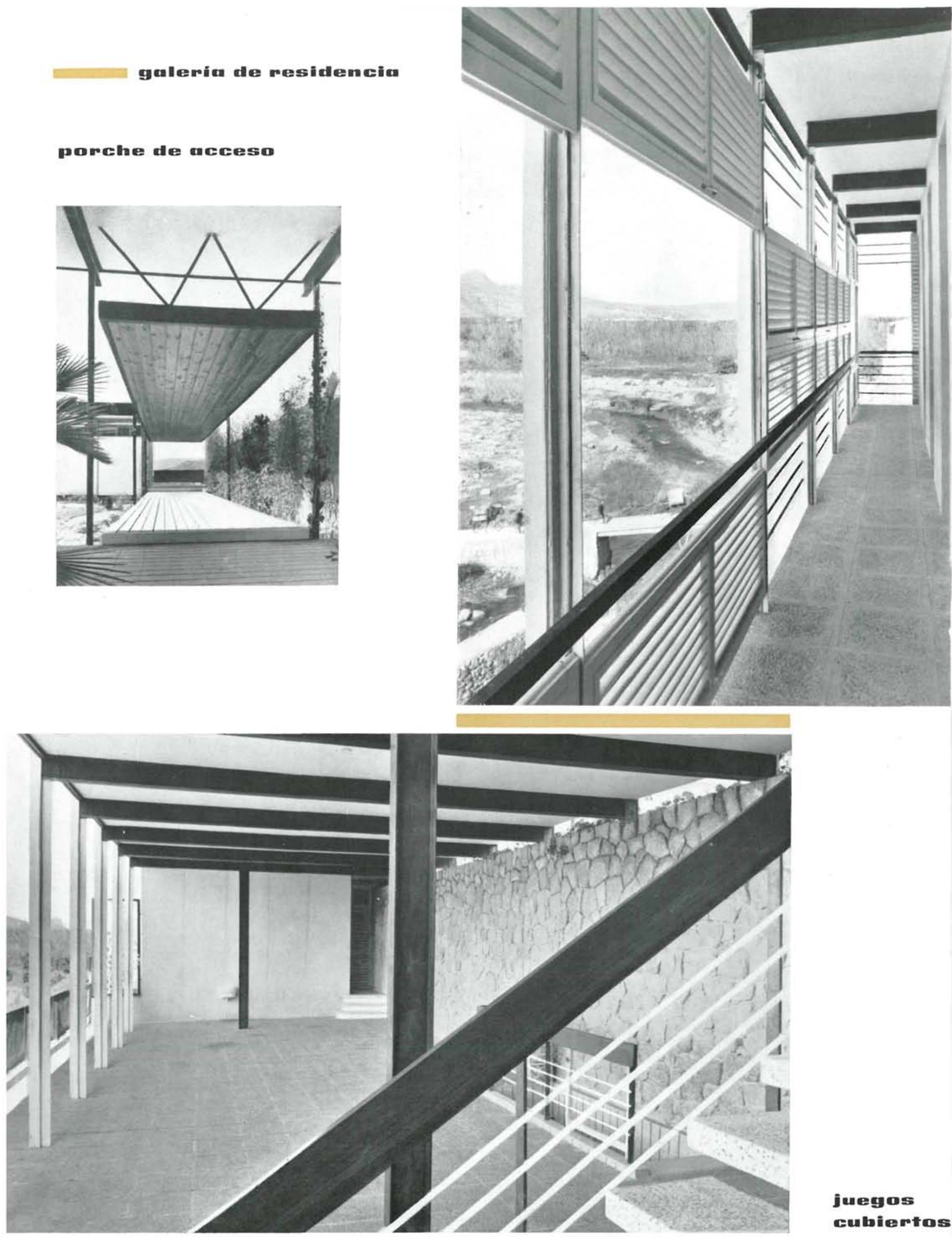


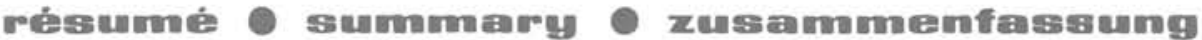

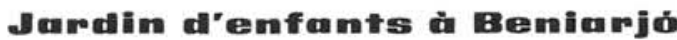

F. M. García Ordóñez et J. M. Dexeus, architectes.

Ce jardin d'enfants a été construit pour que les enfants de Beniarjó puissent être assistés, en l'absence de leurs mères, pendant la cueillette des oranges.

L'édifice se compose des zones suivantes: direction, huit classes, laboratoires, toilettes, terrains de jeux couverts, jardin intérieur, résidence des religieuses et cloître. Chaque zone constitue, dans l'ensemble, un élément bien différencié, grâce à son individualité volumétrique et son caractère.

L'éclairage et la ventilation des diverses dépendances ont été soigneusement étudiés et l'on a réalisé avec bonheur un ensemble très aéré, ainsi qu'à créer une ambiance de grande liberté pour les enfants et à incorporer la beauté du paysage à l'ensemble de l'édifice.

L'ossature est métallique. La brique apparente, ivoire, prédomine dans les classes et un crépi rustique dans le petit couvent.

\section{Ehildrem's mursergy at Bemiarjó}

F. M. García Ordóñez \& J. M. Dexeus, architects.

This children's nursery has been provided so that the children of the town can be looked after during the periods when their mothers are busy collecting oranges.

The building has, in addition to the director's quarters, eight school rooms, wash rooms, covered playing ground, indoor garden, living quarters for the nuns and a cloister. The various functional parts of the building are well differentiated, both spatially and in style.

The lighting and ventilation has been the object of special study, and all the rooms have plenty of light, and are extremely diaphanous. The style aims at giving the children a sense of liberty, and integrating the beauty of the outdoor scenery with the indoor design.

The structure is metallic, and the walls are made of light cream coloured brick in the schoolrooms, whilst the small convent walls have a rustic tyrolean surface.

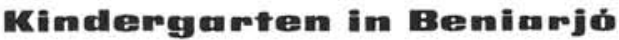

F. M. García Ordóñez und J. M. Dexeus, Architekt.

Dieser Kindergarten wurde zu dem Zweck gebaut, die Kinder von Beniarjó während der Abwesenheit ihrer Mütter in der Zeit der Apfelsinenernte zu versorgen.

Das Gebäude besteht aus folgenden Teilen: Leitung des Kindergartens, 8 Schulzimmern, Laboratorien, Waschräume, überdachter Spieltplatz, Garten, Nonnenwohnheim und Kreuzgang. Jeder dieser einzelnen Teile stellt auf Grund seiner individuellen Raumaufteilung und seines eigenen Charakters ein in sich abgeschlossenes, gut von den anderen zu unterscheidendes Ganzes dar.

Besondere Aufmerksamkeit schenkte man der Beleuchtung und Lüftung der einzelnen Räume und es ist gelungen, dem Gebäude einen Charkter von Durchsichtigkeit zu geben, der den Kindern den Eindruck grosser Weite und Freiheit gibt und gleichzeitig die Schönheit der umgebenden Landschaft mit in das Ganze einbezieht.

Die Struktur des Gebädes besteht aus Metall und es herrscht der mattgelbe Sichtziegelstein in den Schul. zimmern und eine Art Tiroler Verputz in dem kleinen Kloster vor. 